Pacific Journal of Mathematics

MULTIPLICATIVE LINEAR FUNCTIONALS OF STEIN 


\title{
MULTIPLICATIVE LINEAR FUNCTIONALS OF STEIN ALGEBRAS
}

\author{
ROBERT EPHRAIM
}

Let $\left(X, \mathscr{O}_{X}\right)$ be a Stein analytic space, and let $\mathscr{O}(X)$ denote the space of global sections of $\mathcal{O}_{x}$ endowed with its usual Frechet topology. The question of the continuity of complex valued multiplicative linear functionals of $\mathscr{O}(X)$ will be studied. The main result can be stated as follows: Theorem: Let $\left(X, \mathcal{O}_{X}\right)$ be a Stein space, and let $\alpha: \mathcal{O}(X) \rightarrow \mathrm{C}$ be a multiplicative linear functional. Suppose one can find an analytic subset $Y \subset X$ such that all the connected components of both $Y$ and $X-Y$ are finite dimensional. Then $\alpha$ must be continuous. More generally, suppose that one can find a sequence of analytic subsets of $X, X=Y_{0} \supset Y_{1} \supset \cdots \supset$ $Y_{n}=\varnothing$, such that for any $i, 0 \leqq i<n$, all the connected components of $Y_{i}-Y_{i+1}$ are finite dimensional. Then $\alpha$ must be continuous.

This paper resulted from an attempt to understand the claim made without proof in [5] that if $\left(X, \mathscr{O}_{X}\right)$ is a Stein space, and if $\lambda:\left(X, \mathscr{O}_{X}\right) \rightarrow$ Spec $(\mathscr{O}(X))$ is the natural morphism, then the pair $\left(\left(X, \mathcal{O}_{X}\right), \lambda\right)$ is an analytic $C$-cover of $\operatorname{Spec}(\mathcal{O}(X))$. (See [5] for definitions.) In particular, all multiplicative linear functionals of $\mathscr{O}(X)$ would have to be continuous for this to be true. Michael proved the continuity of such functionals in case $X$ is a domain of holomorphy in $C^{n}$ [7]. (He in fact conjectured the continuity of all multiplicative linear functionals on any Frechet algebra [7].) A result of Arens [1] guarantees the desired continuity in case $X$ can be embedded as a closed subspace of some $C^{n}$. Forster [3] proved the desired continuity in case $X$ is finite dimensional. My result is a generalization of Forster's. Markoe [6] gave a weaker extension of Forster's result. He showed continuity under the assumption that $S g(X)$, the singular locus of $X$, is finite dimensional. This follows from my result with $Y_{1}=S g(X)$ and $n=2$. Finally, let me note that an advantage of the techniques of this paper is that they expose the elementary nature of Forster's theorem. They provide a proof which, unlike those in [3] and [6], does not depend on the deep existence of a proper map from a finite dimensional Stein space to some Euclidean space.

1. Preliminaries. Let $X$ be a Stein space. (In what follows I will write $X$ rather than $\left(X, \mathscr{O}_{X}\right)$ for analytic spaces as long as this leads to no ambiguity.) If $\mathscr{F}$ is a coherent analytic sheaf on 
$X$ then $\mathscr{F}(X)$, the space of global sections of $\mathscr{F}$, has a naturally defined Frechet space topology. I will not repeat the definition of that topology here, but I will mention some basic facts about it. (For more details see [2].)

(1.1) If $\mathscr{F} \rightarrow \mathscr{G}$ is a homomorphism of coherent analytic sheaves, then the induced map $\mathscr{F}(X) \rightarrow \mathscr{G}(X)$ is continuous.

(1.2) If $X$ is reduced, then the topology on $\mathscr{O}(X)$ is the topology of uniform convergence on compact subsets of $X$.

We get:

Proposition 1.3. Let $X$ be a Stein space, let $Y$ be any analytic subspace of $X$, and let $r_{X, Y}: \mathscr{O}(X) \rightarrow \mathscr{O}(Y)$ be the canonical restriction map. Then $r_{X, Y}$ is a surjective, continuous, open map.

Proof. The surjectivity follows from Cartan's Theorem B; the continuity follows from (1.1). The openness then follows from the Frechet open mapping theorem.

Corollary 1.4. Let $X$ be a Stein space, and let $Y$ be an analytic subspace of $X$. Suppose $\alpha: \mathcal{O}(X) \rightarrow C$ and $\beta: \mathcal{O}(Y) \rightarrow C$ satisfy $\alpha=\beta \circ r_{X, Y}$. Then $\alpha$ is continuous if and only if $\beta$ is continuous.

Proposition 1.5. Let $X$ be a Stein space and let $X_{\text {red }}$ be its reduction. Then if $\alpha: \mathscr{O}(X) \rightarrow C$ is any multiplicative linear functional, then there is a multiplicative linear functional $\beta: \mathcal{O}\left(X_{r e d}\right) \rightarrow$ $C$ satisfying $\alpha=\beta \circ r_{X, x_{r c d}}$.

Proof. We only need to show that for any $f \in \mathcal{O}(X)$ which is also a section of the nilpotent ideal sheaf of $X$ we have $\alpha(f)=0$. If not, then $g=f-\alpha(f)$ would be a unit in $\mathcal{O}(X)$ satisfying $\alpha(g)=$ 0 . But this would imply $\alpha=0$, a contradiction.

As an immediate consequence of Corollary 1.4 and Proposition 1.5 we get

CoROllary 1.6. Let $X$ be a Stain space. Every multiplicative linear functional on $\mathscr{O}(X)$ is continuous if and only if every multiplicative linear functional on $\mathscr{O}\left(X_{\text {red }}\right)$ is continuous.

This allows for a convenient simplification of the problem. The next result is useful for inductive arguments.

Lemma 1.7. Let $X$ be a Stein space, and let $\alpha: \mathcal{O}(X) \rightarrow C$ be a nonzero multiplicative linear functional. Let $f \in \operatorname{ker} \alpha$. Then 
the coherent ideal sheaf generated by $f$ defines a nonempty Stein subspace $V(f) \subset X$. Moreover, there is a multiplicative linear functional $\beta: \mathcal{O}(V(f)) \rightarrow C$ satisfying $\alpha=\beta \circ r_{X, V(f)}$.

Proof. If $V(f)$ were empty then the germ of $f$ at every point would be a unit, and this would imply that $f$ is a unit in $O(X)$. But then we would have $\alpha=0$, a contradiction.

To prove the existence of $\beta$ we need only show that every section of the coherent ideal sheaf generated by $f$ is an element of ker $\alpha$. But by Cartan's Theorem B every such section is a multiple of $f$ in $\mathscr{O}(X)$, and the result follows.

CoRollary 1.8. Let $X$ be a Stein space. Suppose $X=\Perp X_{i}$, the disjoint union of a family $\left\{X_{i}\right\}_{i \in I}$ of open Stein subspaces of $X$. Then if $\alpha: \mathscr{O}(X) \rightarrow C$ is a multiplicative linear functional there is $a j \in I$ and a multiplicative linear functional $\beta: \mathcal{O}\left(X_{j}\right) \rightarrow C$ satisfying $\alpha=\beta \circ r_{X, x_{j}}$.

Proof. Since $X$ is second countable we may assume that $I$ is a set of integers. We may also assume that $\alpha \neq 0$ since for $\alpha=0$ the result is trivial.

Define $f \in \mathscr{O}(X)$ by $\left.f\right|_{x_{i}}=i$. We have $\alpha(f) \in I$ since otherwise we would have $V(f-\alpha(f))=\varnothing$ contradicting Lemma 1.7. Setting $j=\alpha(f)$ it is clear that $X_{j}=V(f-\alpha(f))$ and the result follows from Lemma 1.7 .

From Corollary 1.4 and Corollary 1.8 we get

Corollary 1.9. Let $X$ be a Stein space. Suppose $X=\Perp X_{i}$, the disjoint union of a family $\left\{X_{i}\right\}_{i \epsilon,}$ of open Stein subspaces of $X$. Then every multiplicative linear functional of $\mathscr{O}(X)$ is continuous if and only if every multiplicative linear functional of $\mathcal{O}\left(X_{i}\right)$ is continuous for all $i \in \mathscr{I}$.

2. Continuity of multiplicative linear functionals, I begin this section by proving Forster's theorem.

Theorem 2.1. Let $X$ be a finite dimensional Stein space. Then every multiplicative linear functional of $\mathcal{O}(X)$ is continuous.

Proof. The proof proceeds by induction on $\operatorname{dim} X$. If $\operatorname{dim} X=$ 0 we use Corollary 1.9 and Corollary 1.6 to reduce to the case $X$ is connected and reduced. But then $X$ is the reduced point and $\mathscr{O}(X)=C$. The result is trivial in this case (since the only multiplicative linear functionals on $C$ are the identity and the zero map). 
Now suppose $\operatorname{dim} X>0$ and that the result has been established for all Stein spaces of dimension $<\operatorname{dim} X$. Again we may assume that $: X$ is connected and reduced. By Cartan's Theorem B we may find an $f \in \mathcal{O}(X)$ which is constant on no irreducible component of $X$. Let $\alpha: \mathscr{O}(X) \rightarrow C$ be a nonzero multiplicative linear functional. Then $f-\alpha(f)$ is not constant on any irreducible component of $X$ so that $\operatorname{dim} V(f-\alpha(f))<\operatorname{dim} X$. Applying Lemma 1.7 we get $\beta$ : $\mathcal{O}(V(f-\alpha(f))) \rightarrow C$ satisfying $\alpha=\beta \circ r_{X, V(f-\alpha(f))}$. It follows from Proposition 1.3 and from the induction hypothesis that $\alpha$ is continuous. This completes the induction step.

\section{From Theorem 2.1 and Corollary 1.9 we get}

COROLlaRy 2.2. Let $X$ be a Stein space and suppose every connected component of $X$ is finite dimensional, then every multiplicative linear functional is continuous.

I now prove my generalization of Forster's theorem.

TheOREm 2.3. Let $X$ be a Stein space. Suppose that one can find a sequence of analytic subsets of $X, X=Y_{0} \supset Y_{1} \supset \cdots \supset Y_{n}=\varnothing$, such that for any $i, 0 \leqq i<n$, all the connected components of $Y_{i}-Y_{i+1}$ are finite dimensional. Then every multiplicative linear functional $\alpha: \mathcal{O}(X) \rightarrow C$ is continuous.

Proof. The proof proceeds by induction on $n$. If $n=1$ then all the connected components of $X$ are finite dimensional and the result follows from Corollary 2.2.

Now suppose $n>1$ and that the result has been established for all Stein spaces admitting the desired type of sequence of analytic subsets, but of length $<n$.

By Corollary 1.6 we may suppose that $X$ and all of the $Y_{i}$ 's have the structure of reduced Stein spaces. If $\alpha(f)=0$ for all $f \in \mathcal{O}(X)$ which vanish on $Y_{1}$ then one can find a $\beta: \mathcal{O}\left(Y_{1}\right) \rightarrow C$ satisfying $\alpha=\beta \circ r_{X, Y_{1}}$. It follows from the induction hypothesis and from Proposition 1.3 that $\alpha$ is continuous in this case.

Otherwise, we can find an $f \in \mathcal{O}(X)$ vanishing on $Y_{1}$ for which $\alpha(f) \neq 0$. Then $V(f-\alpha(f))$ is disjoint from $Y_{1}$. Thus, every connected component of $V(f-\alpha(f))$ is contained in a connected component of $Y_{0}-Y_{1}$, and thus is finite dimensional. By Lemma 1.7 we may find a multiplicative linear functional $\beta: \mathscr{O}(V(f-\alpha(f))) \rightarrow C$ satisfying $\alpha=\beta \circ r_{X \cdot V(f-\alpha(f))}$. It now follows from Corollary 2.2 and Proposition 1.3 that $\alpha$ is continuous in this case as well. This completes the induction step. 


\section{REFERENCES}

1. R. Arens, Dense inverse limit rings, Michigan Math. J., 5 (1958), 169-182.

2. C. Banica, and O. Stanasila, Algebraic Methods in the Global Theory of Complex Spaces, John Wiley \& Sons, New York, 1976.

3. O. Forster, Uniqueness of Topology in Stein Algebras, Function Algebras, F. Birtel, ed., pp. 157-163, Scott Foresman, Chicago, 1966.

4. R. Gunning and H. Rossi, Analytic Functions of Several Complex Variables, Prentice-Hall, Englewood Cliffs, N. J., 1965.

5. H. Hironaka, Resolution of singularities of an algebraic variety over a field of characteristic zero: I, Ann. of Math., (2), 79 (1964), 109-203.

6. A. Markoe, Maximal Ideals of Stein Algebras, Conference on Complex Analysis, edited by W. Zame, pp. 25-39, State University of New York at Buffalo, 1973.

7. E. Michael, Locally multiplicatively convex topological algebras, Mem. Amer. Math. Soc., \#11 (1952).

Received September 13, 1977 and in revised form February 13, 1978.

BRANDEIS UNIVERSITY

WALTHAM, MA 02154 



\section{PACIFIC JOURNAL OF MATHEMATICS}

\section{EDITORS}

RICHARD ARENS (Managing Editor)

University of California

Los Angeles, California 90024

C. W. CurTis

University of Oregon

Eugene, OR 97403

C. C. MOORE

University of California

Berkeley, CA 94720
J. DUGUNDJI

Department of Mathematics University of Southern California Los Angeles, California 90007

R. FinN AND J. Milgram Stanford University Stanford, California 94305

\section{ASSOCIATE EDITORS}

E. F. BECKENBACH

B. H. NeumanN

F. WOLF

K. YoSHIDA

\section{SUPPORTING INSTITUTIONS}

UNIVERSITY OF BRITISH COLUMBIA CALIFORNIA INSTITUTE OF TECHNOLOGY UNIVERSITY OF CALIFORNIA MONTANA STATE UNIVERSITY UNIVERSITY OF NEVADA, RENO NEW MEXICO STATE UNIVERSITY OREGON STATE UNIVERSITY UNIVERSITY OF OREGON
UNIVERSITY OF SOUTHERN CALIFORNIA

STANFORD UNIVERSITY

UNIVERSITY OF HAWAII

UNIVERSITY OF TOKYO

UNIVERSITY OF UTAH

WASHINGTON STATE UNIVERSITY

UNIVERSITY OF WASHINGTON 


\section{Pacific Journal of Mathematics \\ Vol. 78, No. $1 \quad$ March, 1978}

Simeon M. Berman, A class of isotropic distributions in $\mathbf{R}^{n}$ and their

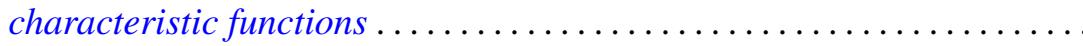

Ezra Brown and Charles John Parry, The 2-class group of biquadratic fields.

II ........................................ 11

Thomas E. Cecil and Patrick J. Ryan, Focal sets of submanifolds ....... 27

Joseph A. Cima and James Warren Roberts, Denting points in $B^{p} \ldots \ldots \ldots 41$

Thomas W. Cusick, Integer multiples of periodic continued fractions . . . . . 47

Robert D. Davis, The factors of the ramification sequence of a class of

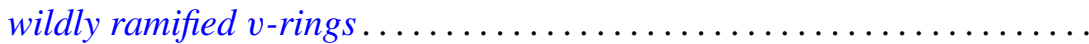

Robert Martin Ephraim, Multiplicative linear functionals of Stein

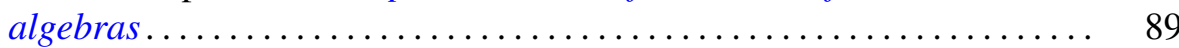

Philip Joel Feinsilver, Operator calculus . .................... 95

David Andrew Gay and William Yslas Vélez, On the degree of the splitting field of an irreducible binomial ..........................

Robert William Gilmer, Jr. and William James Heinzer, On the divisors of

monic polynomials over a commutative ring ..................

Robert E. Hartwig, Schur's theorem and the Drazin inverse .............

Hugh M. Hilden, Embeddings and branched covering spaces for three and four dimensional manifolds ............................

Carlos Moreno, The Petersson inner product and the residue of an Euler product. ...

Christopher Lloyd Morgan, On relations for representations of finite groups....

Ira J. Papick, Finite type extensions and coherence

$\mathrm{R}$. Michael Range, The Carathéodory metric and holomorphic maps on a class of weakly pseudoconvex domains ................

Donald Michael Redmond, Mean value theorems for a class of Dirichlet series

Daniel Reich, Partitioning integers using a finitely generated semigroup ...

Georg Johann Rieger, Remark on a paper of Stux concerning squarefree

numbers in non-linear sequences

Gerhard Rosenberger, Alternierende Produkte in freien Gruppen ..

Ryōtarō Satō, Contraction semigroups in Lebesgue space

Tord Sjödin, Capacities of compact sets in linear subspaces of $\mathbf{R}^{n}$

Robert Jeffrey Zimmer, Uniform subgroups and ergodic actions of exponential Lie groups......................... 